\title{
Das Anna-Offizium des Anna Hannsen Schuman-Kodex
}

\author{
Ilona FERENCZI \\ Institut für Musikwissenschaft \\ Forschungszentrum für Humanwissenschaften \\ Ungarische Akademie der Wissenschaften \\ Táncsics Mihály u. 7, H-1014 Budapest, Ungarn \\ E-Mail: ferenczi.ilona@btk.mta.hu
}

(Angekommen: Februar 2015; angenommen: April 2015)

\begin{abstract}
Only two of the five polyphonic settings of St Anne's liturgy in the 16thcentury Vesperale Anna Hannsen Schuman at the Slovenský Národný Archív in Bratislava are correctly texted. This paper shows how the rhymed responsory Iesu Christe nepos cuius tu could be identified and the texts of the rhymed antiphons $O$ beata Christi ava and Annae sanctae celebremus complemented by consulting a plainchant source from Kirnberg an der Mank in lower Austria.
\end{abstract}

Keywords: St Anne Officium, Vesperale, Kodex Anna Hannsen Schuman, Kirnberg antiphonal

Meine fachliche Beschäftigung mit dem Kodex Anna Hannsen Schuman begann vor über vierzig Jahren. ${ }^{1}$ Als bisher einziges umfangreiches, wenn auch nicht ganz fehlerfreies Ergebnis meiner Untersuchungen erschien 1975 eine hundert Seiten starke Beschreibung des Inhalts des Kodex in dieser Zeitschrift. ${ }^{2}$ Darin konnte ich mit der unschätzbaren Hilfe von László Dobszay die liturgische Gattung der meisten vollständigen bzw. fragmentarischen Sätze bestimmen und sie damit in die liturgische Ordnung einfügen. Natürlich ergaben sich dabei Schwierigkeiten, die einer vollständigen Einordnung der Sätze im Wege standen, wie z. B. in Fällen, in denen das mehrstimmig nur mit Versus und Repetenda notierte gregorianische „Responsorium“ nicht in den ungarischen mittelalterlichen Quellen zu finden

1. Die endgültige Benennung des Kodex siehe später.

2. Ilona Ferenczi, „Mehrstimmige Sammlung aus dem 16. Jahrhundert in Pressburg (Kodex Anna Hannsen Schuman)“, Studia Musicologica 17 (1975), 59-165. 
war. So kam es vor, dass der Titel des ganzen Satzes manchmal nicht bestimmt werden konnte, nur der darin enthaltene Versus. Dies war auch der Fall beim auf fol. 142v-144r notierten Versus Fecundatos, zu dessen Repetenda im Kodex der Text Regno trans hinzugefügt wurde. Zudem waren nur die Textanfänge und die unvollständige Doxologie eingetragen und auch sie ausschließlich in der Tenorstimme. Dank der im Internet zugänglichen Cantus-Database ist mir inzwischen gelangen, dieses Responsorium zu identifizieren. Die Cantus-Database half auch bei der Bestimmung der dem Responsorium folgenden Antiphon, deren Textanfang im Kodex fehlerhaft angegeben ist, sowie bei der Identifizierung der ihm vorangehenden Antiphon, des einzigen völlig textlos gebliebenen Satzes im Kodex. Im Folgenden möchte ich das Ergebnis des Identifizierungsprozesses vorlegen.

\section{$* * *$}

Der Anna Hannsen Schuman-Kodex kam 1571 in den Besitz des Pressburger Domkapitels St. Martin, also bereits zu der Zeit, als statt des von den Osmanen besetzten historischen Sitzes des Erzbischofs das Zentrum des Erzbistums Esztergom/Gran nach Tyrnau (heute Trnava in der Slowakei) bzw. Pressburg (Bratislava) versetzt worden war. Die Benennung des Kodex stammt von dem Schenkungstext auf der Innenseite des Einbandes: Anna Weilendt Hannsen Schuman gewesnem Burger alhie zu Preßburg verlaßnen Wittib schenkt dieses buech in die khirchen alhie zu sanndt Mertn zu ainer gedechtnus. Geschehen in funftzehen hundert und Ainundsibentzigistn Jar.

Der Kodex wurde vier Jahrhunderte hindurch im Kapitel aufbewahrt; in dem von Nándor Knauz zusammengestellten Katalog der Handschriften des Kapitels trägt er die Signatur Knauz 11. ${ }^{3} 1972$ wurde er restauriert und kam dann 1983 an seinen derzeitigen Bewahrungsort, ins Slowakische Staatsarchiv in Pressburg/ Bratislava. ${ }^{4}$ Außer der Schenkung und dem Fundort wird der Kodex mit Pressburg durch die nachträglich eingetragenen Texte verbunden, die einen lokalen Gebrauch wahrscheinlich werden lassen. Die Klärung der Entstehungsumstände und der Herkunft des Kodex ist noch nicht abgeschlossen. ${ }^{5}$

Der Kodex ist eine Art polyphones Vesperale. Von den 244 darin enthaltenen lateinischen liturgischen Sätzen bzw. Kompositionen gehören nur sieben nicht zur Vesperliturgie. Beinahe die Hälfte sind Vertonungen von Antiphonen, die andere Hälfte besteht aus Responsorien, Hymnen und Magnifikats, ergänzt durch sonstige Vespersätze wie Invocatio, Versikel, Benedicamus und mehrstimmiges Rezi-

3. Nándor Knauz, A pozsonyi káptalannak kéziratai [Handschriften des Pressburger Domkapitels] (Esztergom: Horák, [1870]).

4. Bratislava, Slovenský Národný Archív.

5. Die meisten Wasserzeichen des Kodex weisen auf Prag als Herkunftsort hin. Vgl. Charles M. Briquet, Les Filigranes. Dictionnaire historique des marques du papier, dès leur apparition vers 1282 jusqu'en 1600 (Genève-Paris: Alphonse Picard et fils, 1907), Nr. 495, 762, 1012. 
tieren der Psalmen. In der Geschichte der mittel-, ja sogar der gesamteuropäischen Musik kam es selten vor, dass für die ganzjährige Vesperliturgie, also nicht nur für die wichtigeren Feste, mehrstimmige Satzserien zusammengestellt wurden. ${ }^{6}$ Da es sich bei den Stücken nicht um selbstständige Motetten, sondern um eine die Vesperliturgie bereichernde Musik, ja manchmal sogar um nur teilweise durchkomponierte Sätze handelt, wurden die Werke allgemein unter denen geringeren Umfanges ausgewählt und nur einige besser ausgearbeitete Kompositionen mit aufgenommen. Mit Verfassernamen sind nur zwei Sätze versehen, und zwar mit dem Namen von Mouton. ${ }^{7}$ Bei der Durchsicht anderer zeitgenössischer Sammlungen konnten jedoch weitere Komponisten wie Josquin, Walter, Finck, Dietrich, Reneri, Morales, Mahu, Divitis und Richaforti identifiziert werden. Die Liste der Komponisten legt die Annahme nahe, dass, obwohl der Kodex erst 1571 dem Domkapitel St. Martin geschenkt wurde, die in ihm enthaltenen Sätze einige Jahrzehnte früher entstanden sind: Wahrscheinlich wurden sie in den 1540er Jahren aus verschiedenen Handschriften und Drucken mit deutschem Bezug ausgesucht. Bei liturgischen Sätzen kürzeren Umfanges, vor allem bei Antiphonen und Responsorien, konnte die Verfasserschaft nicht immer festgestellt werden. Zu dieser Gruppe gehören auch die Kompositionen der St. Anna-Liturgie.

In den letzten Jahren wurde von mir die Publikation des ganzen Anna Hannsen Schuman-Kodex in der Serie Musicalia Danubiana des Instituts für Musikwissenschaft der Ungarischen Akademie der Wissenschaften in Angriff genommen. Bei den Vorarbeiten zur Veröffentlichung bereitete die Zusammenstellung des St. Anna-Offiziums die meisten Probleme: Die fehlerhaft textierten oder gar textlosen mehrstimmigen Sätze erschwerten die Ausgestaltung des vollständigen Offiziums. Das Anna-Offizium beginnt auf fol. 140 des Kodex, wo der Schreiber die Bestimmung des Festes mit der Eintragung Anne angab. Den Ausgangspunkt der Serie bildet die vierstimmige Vertonung der Antiphon Celeste beneficium. Daran schließt sich das Responsorium Firma fide fidens an, von dem nur Versus und Repetenda mehrstimmig notiert wurden (Quia et Deus; Cum presertim Annae). Bei dem darauffolgenden Satz fehlt jeglicher Text, so dass der Eindruck entsteht, als wenn hier die liturgische Ordnung unterbrochen wäre. Dass das Stück doch zur St. Anna-Liturgie gehört, lassen beide nachfolgenden Sätze vermuten, deren Texte mit Hilfe der Cantus-Database identifiziert werden konnten. In einem aus dem niederösterreichischen Kirnberg stammenden, an der Wende zum

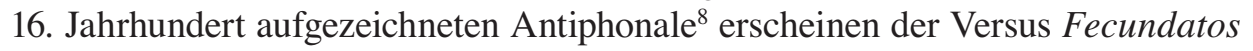
und die Repetenda Regno trans als Bestandteile des Responsoriums Iesu Chris-

6. Dazu siehe z. B. David E. Crawford, Vespers Polyphony at Modena's Cathedral in the First Half of the Sixteenth Century (Ph. D. diss., University of Illinois, 1967) - anhand 13 hierzu gehörender Quellen.

7. Fol. 11v-15r Hodie Christus natus est, fol. 369v-380r Magnifikat im 6. Ton.

8. Wien, Diözesanarchiv, D-4. 
te nepos cuius tu. ${ }^{9}$ Nach dem Responsorium folgt - wie in dem Anna Hannsen Schuman-Kodex - auch im Kirnberger Antiphonale eine Antiphon. Während bei der Cantus-Stimme der mehrstimmigen Bearbeitung nur die erste Textzeile ausgeschrieben ist ( $O$ beata Christi $\underline{\text { Anna) }}$ ), findet sich im Kirnberger Antiphonale bei der dem Magnifikat zugeordneten Antiphon im 5. Modus der ganze Text, und zwar mit der richtigen Anfangszeile O beata Christi ava (O, Christi selige Großmutter). Mit Ausnahme der ersten Cantus-Zeile hat man bei den textlos belassenen Stimmen später - wahrscheinlich schon in Pressburg - in kursiver Schrift den Text der Antiphon für das St. Elisabeth-Fest eingetragen: Exultet vox Ecclesiae. ${ }^{10}$ Zwar ist der Text der ursprünglich im 4. Modus verfassten Elisabeth-Antiphon wesentlich länger, er ließ sich den melismatischen Passagen der für den Anna-Text geschriebenen mehrstimmigen Bearbeitung leicht anpassen. ${ }^{11}$

Von den vermutlich fünf Sätzen des St. Anna-Offiziums im Anna Hannsen Schuman-Kodex war damit die Textfrage bei vieren geklärt, und nur die des völlig textlosen mittleren Stücks blieb ungelöst. Jedoch dadurch, dass die Zugehörigkeit zu dem Festkreis von beiden Sätzen sowohl vor wie nach dem textlosen Satz erwiesen werden konnte, wurde auch sein Bezug zum St. Anna-Fest wahrscheinlich gemacht - umso mehr, als sein Cantus firmus formal eindeutig auf die Antiphon-Gattung hinwies. Zwar kommt die Melodie in der Antiphonen-Gesamtausgabe der Serie Monumenta Monodica Medii Aevi unter den Antiphonen im 1. Modus nicht vor, die Kirnberger Antiphonale jedoch bringt sie am Anfang der St. Anna-Liturgie mit dem Text Annae sanctae celebremus auf derselben Seite wie das Responsorium Iesu Christe nepos cuius tu (Notenbeispiel 1). ${ }^{12}$

Notenbeispiel 1 Antiphon Annae sanctae celebremus

Wien, Diözesanarchiv, D-4, fol. 230v, neulich fol. 223v

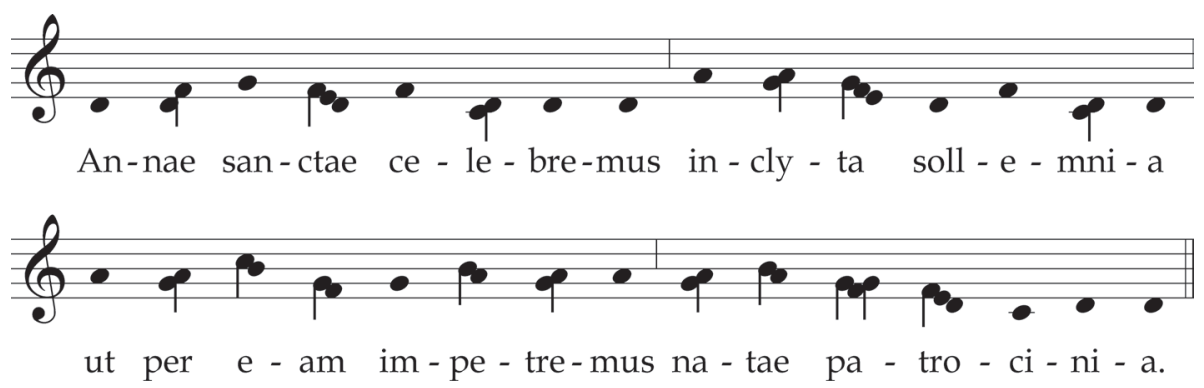

9. Der ursprünglichen Folienzählung des Antiphonals nach fol. 230v, der neueren gemäß fol. 223v.

10. Vgl. Antiphonen, hrsg. von László Dobszay und Janka Szendrei (Kassel etc.: Bärenreiter, 1999) (=Monumenta Monodica Medii Aevi 5 [im Weiteren: MMMAe]), Nr. 4328.

11. Die gereimte Antiphon O beata Christi ava hat 39 Silben $(8,8,8,8$, 7), die ebenfalls gereimte Exultet vox Ecclesiae dagegen 61 Silben (8, 7, 7, 6, 6, 7, 7, 7, 6).

12. Unter den 602 Antiphonen im 1. Modus der ungarischen Antiphonen-Gesamtausgabe fanden wir nur eine einzige, die in Form, Melodieführung und Kadenz zur Anna-Antiphon zu stellen ist: die Antiphon Laetare Germania, vgl. MMMAe Nr. 1545. 
Demnach steht das St. Anna-Offizium im Pressburger Anna Hannsen Schuman-Kodex so vor uns:

fol. 139v-140r - Caeleste beneficium - Antiphon im 1. Modus

fol. 140v-142r - Firma fide fidens - Versus und Repetenda vom Responsorium im 3. Modus

fol. $141 \mathrm{v}-143 \mathrm{r}$ - Annae sanctae celebremus - Antiphon im 1. Modus

fol. 142v-144r - Iesu Christe nepos cuius tu - Versus und Repetenda vom Responsorium im 5. Modus

fol. $143 \mathrm{v}-145 \mathrm{r}-\mathrm{O}$ beata Christi ava - Antiphon im 5. Modus

Aufgrund dieses Fundes konnten die Stimmen der Antiphonen-Bearbeitungen des St. Anna-Offiziums für die geplante Publikation mit Text versehen werden. Bei dem textlosen Satz wurde das Vorhaben dadurch erleichtert, dass die vierstimmige Bearbeitung ersichtlich bestrebt ist, der Melodie, Gliederung und Form des verwendeten gregorianischen Musters zu folgen. Dabei sind die zweimal zwei Zeilen der Antiphon in der mehrstimmigen Bearbeitung nicht proportional verteilt: Der erste Teil des Chorals reicht vom ersten bis zum dreizehnten Takt, der zweite vom dreizehnten bis zum zweiunddreißigsten, wobei sich die letzte Melodiezeile weiter ausbreitet. Die vier Melodiezeilen der Antiphon werden durch die Tenor- und die Cantus-Stimme einmal streng und ein andermal ungenau imitierend dargeboten. Die dritte Melodiezeile wird durch die Tenorstimme sogar mit einem breiteren Melodiebogen als die originale Antiphon nachgezeichnet. Der besondere Klang des Satzes ergibt sich daraus, dass zwischen dem Cantus, der die Melodie der Antiphon im 1. Modus vorträgt, und der den Cantus mehrmals vorimitierenden Tenorstimme keine wirkliche Alt-, sondern eine Contratenor-Stimme liegt und dass diese - gewissermaßen auf die Technik des 15. Jahrhunderts rückverweisende - Dreistimmigkeit durch eine Bassstimme bereichert wird. An einer Stelle wird die in der Vierstimmigkeit verborgene Dreistimmigkeit offensichtlich: Der von Takt 27 auf Takt 28 auftretenden unvermeidlichen Quintparallele zwischen Cantus und Bass folgt eine Primparallele zwischen Bass- und Tenorstimme, d. h. für einen Moment wird das musikalische Material dreistimmig, dann bewegt es sich in Sextakkord-Parallelen in Fauxbourdon-Technik fort (Abbildung 1-2, Notenbeispiel 2).

Zusammenfassend kann man festhalten, dass die St. Anna-Liturgie zu den weniger ausgearbeiteten Teilen des Anna Hannsen Schuman-Kodex gehört. Von fünf einander folgenden Sätzen wurden vom Versus und der Repetenda eines Responsoriums nur die ersten Worte notiert, von einer Antiphon ebenfalls nur der Anfang - und auch der fehlerhaft -, eine andere Antiphon blieb ganz ohne Text. Die „unfertige“ Gestalt der Anna-Sätze lässt die Hypothese zu, dass sie am Ort 
der Herstellung des Kodex komponiert wurden und zur Zeit ihrer Eintragung ihre Komposition noch nicht abgeschlossen war.

Bei aller Mangelhaftigkeit in der Ausarbeitung des Offiziums kann man doch nicht behaupten, dass das St. Anna-Fest im Kodex vernachlässigt wurde. In der Hymnenserie erscheint der Text des Anna-Satzes Ex hac carnis sancta als Bearbeitung der Melodie Ave maris stella, deren erste Strophe Lucis huius festa ist. ${ }^{13}$ Auch hierbei gab man - wie allgemein in den meisten damaligen Hymnenbearbeitungen - die Texte der geraden Strophen an, da die ungeraden einstimmig mit gregorianischen Melodien gesungen wurden. Unvermutet erscheint St. Anna auch in der die Hl. Katharina beehrende Antiphon Ave gemma claritatis: Die Stelle mit dem Namen der Gefeierten enthält eine für das Fest der Anna wählbare Alternative: ${ }^{14}$

Ave gemma claritatis ad instar carbunculi

ave rosa paradisi more fragrans balsami

Catherina virgo felix / gloriosa meritis

Anna mater matris Christi / gloriosa meritis

assistentes tuis festis caeli iunge gaudiis.

14. Fol. 245v-247r. - Angemerkt sei, dass die Antiphonen-Gesamtausgabe die Antiphon für zwei verschiedene Feste, in zwei Tönen und zwei kaum voneinander abweichenden Textvarianten veröffentlicht: Nr. 1598 für das Fest der Katharina - auch der Satz im AHS-Kodex beruht darauf - und Nr. 3179 für das der Anna. In die Cantus-Database gelangten diese Graner Quellen nicht. 
AbbiLdung 1-2 Vierstimmiger Satz ohne Text im Anna Hannsen Schuman-Kodex

Pressburg/Bratislava Slovenský Národný Archív, Knauz 11, fol. 141v-143r

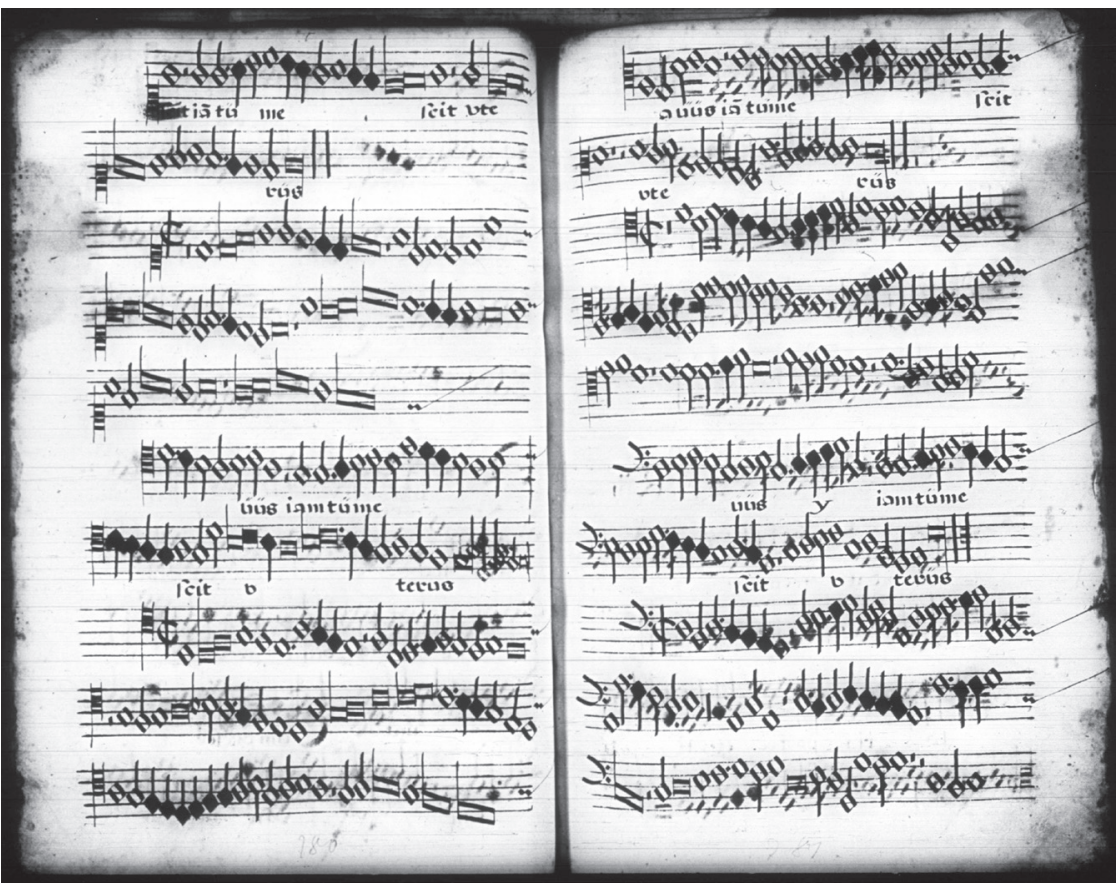

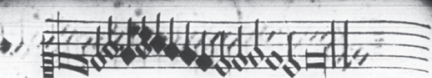

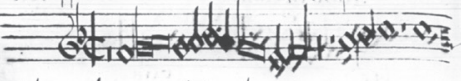
. . . 2. Rii.

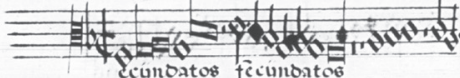

1. (1)

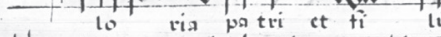
+
10 1.

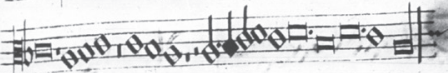
等 $\Longrightarrow+200$ Donose

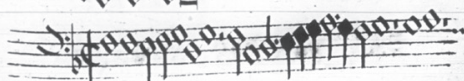

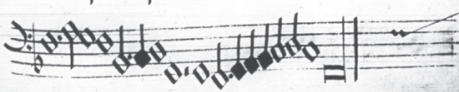


NotenbeISPIEl 2 Übertragung des mehrstimmigen Satzes aus dem Anna Hannsen Schuman-

Kodex, fol. 141v-143r mit Textergänzung der Antiphon Annae sanctae celebremus

\section{Annae sanctae celebremus}
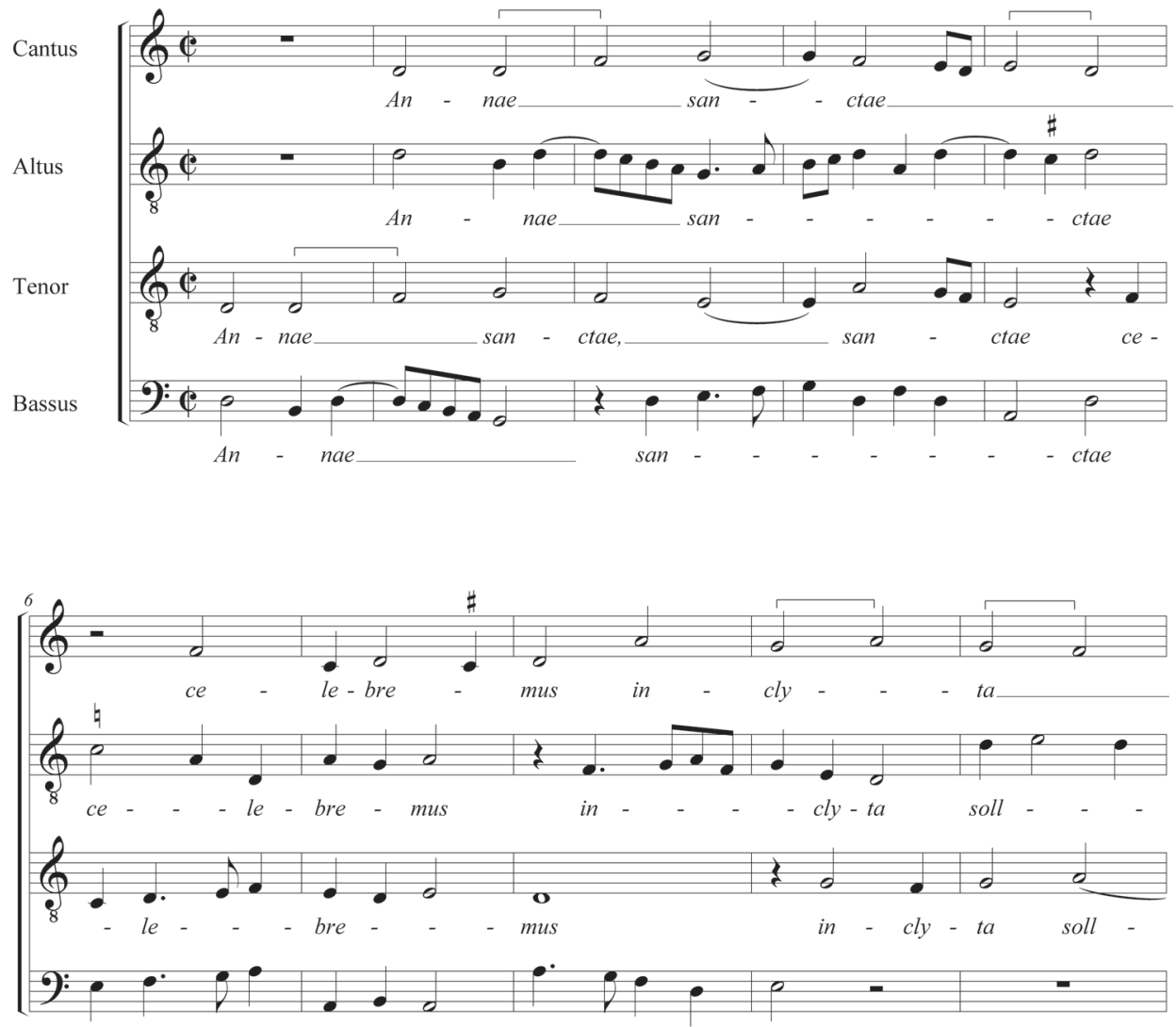

ce-le - - - bre - mus, ce - le-bre - mus

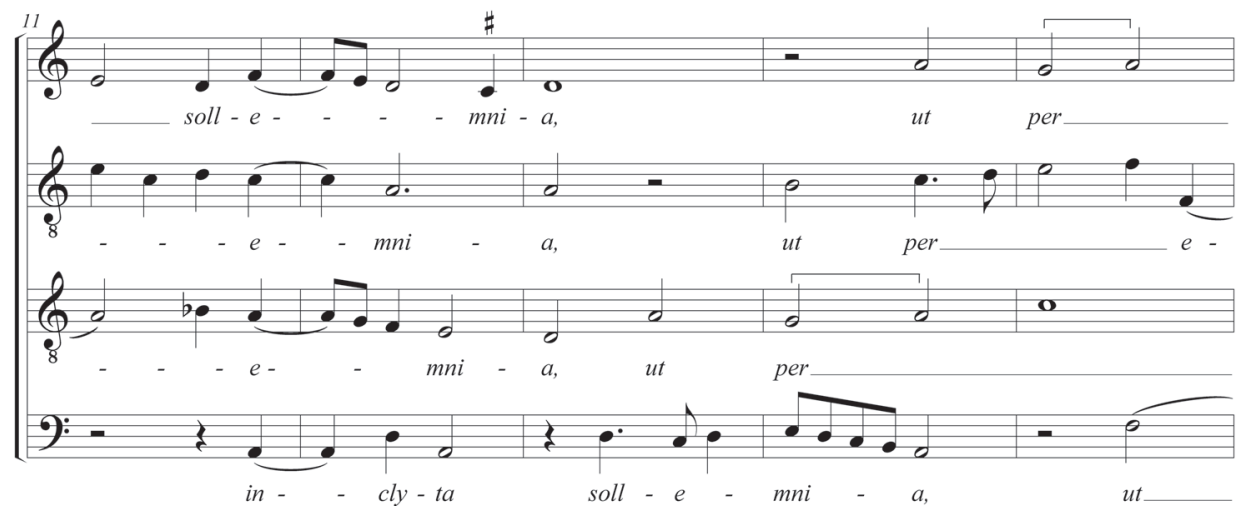

stuaı Musıcologıca $\mathrm{in}, \angle \bar{U} 1 \mathrm{~J}$

$l y-t a$

soll $-e-m n i$

ut 

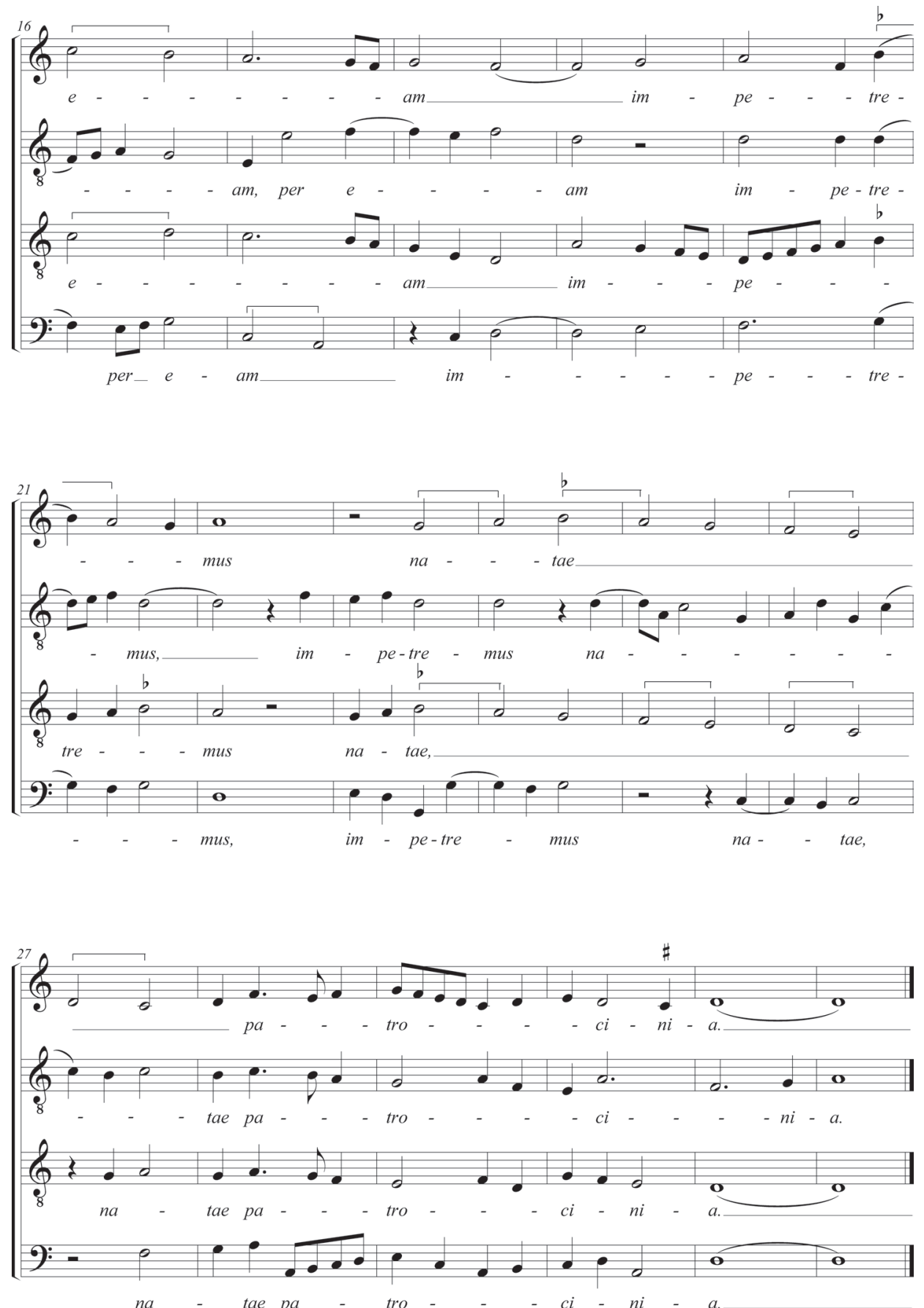